Article

\title{
Higher-Order Convolutions for Apostol-Bernoulli, Apostol-Euler and Apostol-Genocchi Polynomials
}

\author{
Yuan He ${ }^{1} \mathbb{D}$, Serkan Araci ${ }^{2, *(\mathbb{D})}$, Hari M. Srivastava ${ }^{3,4} \mathbb{D}$ and Mahmoud Abdel-Aty ${ }^{5}$ \\ 1 Faculty of Science, Kunming University of Science and Technology, Kunming 650500, Yunnan, China; \\ hyyhe@aliyun.com or hyyhe@outlook.com \\ 2 Faculty of Economics, Administrative and Social Sciences, Hasan Kalyoncu University, \\ Gaziantep TR-27410, Turkey \\ 3 Department of Mathematics and Statistics, University of Victoria, Victoria, BC V8W 3R4, Canada; \\ harimsri@math.uvic.ca \\ 4 Department of Medical Research, China Medical University, Taichung 40402, Taiwan \\ 5 Zewail City of Science andTechnology, University of Science and Technology, Cairo 82524, Egypt; \\ amisaty@gmail.com \\ * Correspondence: serkan.araci@hku.edu.tr or mtsrkn@hotmail.com
}

Received: 20 November 2018; Accepted: 10 December 2018; Published: 14 December 2018

check for updates

\begin{abstract}
In this paper, we present a systematic and unified investigation for the Apostol-Bernoulli polynomials, the Apostol-Euler polynomials and the Apostol-Genocchi polynomials. By applying the generating-function methods and summation-transform techniques, we establish some higher-order convolutions for the Apostol-Bernoulli polynomials, the Apostol-Euler polynomials and the Apostol-Genocchi polynomials. Some results presented here are the corresponding extensions of several known formulas.
\end{abstract}

Keywords: Apostol-Bernoulli polynomials; Apostol-Euler polynomials; Apostol-Genocchi polynomials; convolution identities; stirling numbers of the first and second kind

\section{Introduction}

Throughout this paper, $\mathbb{C}$ and $\mathbb{C}^{\times}$denote the set of complex numbers and the set of complex numbers excluding zero, respectively. We also denote by $\mathbb{N}$ and $\mathbb{N}^{*}$ the set of positive integers and the set of non-negative integers, respectively. For $\alpha, \lambda \in \mathbb{C}$, the generalized Apostol-Bernoulli polynomials $\mathcal{B}_{n}^{(\alpha)}(x ; \lambda)$, the generalized Apostol-Euler polynomials $\mathcal{E}_{n}^{(\alpha)}(x ; \lambda)$ and the generalized Apostol-Genocchi polynomials $\mathcal{G}_{n}^{(\alpha)}(x ; \lambda)$ of order $\alpha$ are defined by the following generating functions (see, e.g., [1-4]):

$$
\begin{gathered}
\left(\frac{t}{\lambda e^{t}-1}\right)^{\alpha} e^{x t}=\sum_{n=0}^{\infty} \mathcal{B}_{n}^{(\alpha)}(x ; \lambda) \frac{t^{n}}{n !} \\
\left(|t|<2 \pi \text { when } \lambda=1 ;|t|<|\log \lambda| \text { when } \lambda \neq 1 ; 1^{\alpha}:=1\right), \\
\left(\frac{2}{\lambda e^{t}+1}\right)^{\alpha} e^{x t}=\sum_{n=0}^{\infty} \mathcal{E}_{n}^{(\alpha)}(x ; \lambda) \frac{t^{n}}{n !} \\
\left(|t|<\pi \text { when } \lambda=1 ;|t|<|\log (-\lambda)| \text { when } \lambda \neq 1 ; 1^{\alpha}:=1\right)
\end{gathered}
$$

and

$$
\begin{gathered}
\left(\frac{2 t}{\lambda e^{t}+1}\right)^{\alpha} e^{x t}=\sum_{n=0}^{\infty} \mathcal{G}_{n}^{(\alpha)}(x ; \lambda) \frac{t^{n}}{n !} \\
\left(|t|<\pi \text { when } \lambda=1 ;|t|<|\log (-\lambda)| \text { when } \lambda \neq 1 ; 1^{\alpha}:=1\right) .
\end{gathered}
$$


In particular, the polynomials $\mathcal{B}_{n}(x ; \lambda), \mathcal{E}_{n}(x ; \lambda)$ and $\mathcal{G}_{n}(x ; \lambda)$ given by

$$
\mathcal{B}_{n}(x ; \lambda)=\mathcal{B}_{n}^{(1)}(x ; \lambda), \quad \mathcal{E}_{n}(x ; \lambda)=\mathcal{E}_{n}^{(1)}(x ; \lambda)
$$

and

$$
\mathcal{G}_{n}(x ; \lambda)=\mathcal{G}_{n}^{(1)}(x ; \lambda)
$$

are called the Apostol-Bernoulli polynomials, the Apostol-Euler polynomials and the Apostol-Genocchi polynomials, respectively. The Apostol-Bernoulli numbers $\mathcal{B}_{n}(\lambda)$, the Apostol-Euler numbers $\mathcal{E}_{n}(\lambda)$ and the Apostol-Genocchi numbers $\mathcal{G}_{n}(\lambda)$ are expressed by means of the Apostol-Bernoulli polynomials, the Apostol-Euler polynomials and the Apostol-Genocchi polynomials, as follows:

$$
\mathcal{B}_{n}(\lambda)=\mathcal{B}_{n}(0 ; \lambda), \quad \mathcal{E}_{n}(\lambda)=2^{n} \mathcal{E}_{n}\left(\frac{1}{2} ; \lambda\right) \quad \text { and } \quad \mathcal{G}_{n}(\lambda)=\mathcal{G}_{n}(0 ; \lambda) .
$$

Furthermore, the case $\alpha=\lambda=1$ in (1), (2) and (3) gives the Bernoulli polynomials $B_{n}(x)$, the Euler polynomials $E_{n}(x)$ and the Genocchi polynomials $G_{n}(x)$, that is,

$$
B_{n}(x)=\mathcal{B}_{n}^{(1)}(x ; 1), \quad E_{n}(x)=\mathcal{E}_{n}^{(1)}(x ; 1) \quad \text { and } \quad G_{n}(x)=\mathcal{G}_{n}^{(1)}(x ; 1) .
$$

Also the case $\lambda=1$ in (4) gives the Bernoulli numbers $B_{n}$, the Euler numbers $E_{n}$ and the Genocchi numbers $G_{n}$ as follows:

$$
B_{n}=B_{n}(0), \quad E_{n}=2^{n} E_{n}\left(\frac{1}{2}\right) \quad \text { and } \quad G_{n}=G_{n}(0) .
$$

Recently, the above-defined generalized Apostol-Bernoulli polynomials, the generalized Apostol-Euler polynomials and the generalized Apostol-Genocchi polynomials was unified by the following generating function (see, for example, [5]):

$$
\begin{gathered}
\left(\frac{2^{1-\kappa} t^{\kappa}}{\beta^{b} e^{t}-a^{b}}\right)^{\alpha} e^{x t}=\sum_{n=0}^{\infty} \mathcal{Y}_{n, \beta}^{(\alpha)}(x ; \kappa, a, b) \frac{t^{n}}{n !} \\
\left(|t|<2 \pi \text { when } \beta=a ;|t|<\log \left(\frac{\beta}{a}\right) \text { when } \beta \neq a ;\right. \\
\left.\kappa, \beta \in \mathbb{C} ; a, b \in \mathbb{C}^{\times} ; 1^{\alpha}:=1\right) .
\end{gathered}
$$

It is worth mentioning that the case $\alpha=1$ in (5) was constructed by Ozden et al. [6,7]. It is easily seen that the polynomials $\mathcal{Y}_{n, \beta}(x ; \kappa, a, b)$ given by

$$
\mathcal{Y}_{n, \beta}(x ; \kappa, a, b)=\mathcal{Y}_{n, \beta}^{(1)}(x ; \kappa, a, b)
$$

can be regarded as a generalization and unification of the Apostol-Bernoulli polynomials, the Apostol-Euler polynomials and the Apostol-Genocchi polynomials with, of course, suitable choices of the parameter $a, b$ and $\beta$. We refer to the recent works [8-13] on these Apostol-type polynomials and numbers.

In the present paper, we shall be concerned with some higher-order convolutions for the Apostol-Bernoulli polynomials, the Apostol-Euler polynomials and the Apostol-Genocchi polynomials. The idea stems from the higher-order convolutions for the Bernoulli polynomials due to Agoh and Dilcher [14], Bayad and Kim [15] and Bayad and Komatsu [16]. We establish several 
higher-order convolutions for the Apostol-Bernoulli polynomials, the Apostol-Euler polynomials and the Apostol-Genocchi polynomials by making use of the generating-function methods and summation-transform techniques. It turns out that several interesting known results are obtainable as special cases of our main results.

This paper is organized as follows. In Section 2, we first give the higher-order convolution for the polynomials defined by (5) $\mathcal{Y}_{n, \beta}(x ; \kappa, a, b)$ and then present the corresponding higher-order convolutions for the Apostol-Bernoulli polynomials, the Apostol-Euler polynomials and the Apostol-Genocchi polynomials. Moreover, several corollaries and consequences of our main theorems are also deduced. Section 3 is devoted to the proofs of the main results by applying the generating-function methods and summation-transform techniques.

\section{Main Results}

As usual, by $\left(\begin{array}{l}\lambda \\ n\end{array}\right)$ we denote the binomial coefficients given, for $\lambda \in \mathbb{C}$, by

$$
\left(\begin{array}{l}
\lambda \\
0
\end{array}\right)=1 \text { and }\left(\begin{array}{l}
\lambda \\
n
\end{array}\right)=\frac{\lambda(\lambda-1) \cdots(\lambda-n+1)}{n !} \quad(n \in \mathbb{N}) .
$$

The multinomial coefficient

$$
\left(\begin{array}{c}
n \\
r_{1}, \cdots, r_{k}
\end{array}\right)
$$

is given, for $n, r_{1}, \cdots, r_{k} \in \mathbb{N}^{*} \quad(k \in \mathbb{N})$, by

$$
\left(\begin{array}{c}
n \\
r_{1}, \cdots, r_{k}
\end{array}\right)=\frac{n !}{r_{1} ! \cdots r_{k} !} \quad(k \in \mathbb{N}) .
$$

We also denote by $s(n, k)$ the Stirling numbers of the first kind and by $S(n, k)$ the Stirling numbers of the second kind, which are usually defined by the following generating functions (see, for example, $[17,18])$ :

$$
\frac{[\ln (1+t)]^{k}}{k !}=\sum_{n=k}^{\infty} s(n, k) \frac{t^{n}}{n !} \quad \text { and } \quad \frac{\left(e^{t}-1\right)^{k}}{k !}=\sum_{n=k}^{\infty} S(n, k) \frac{t^{n}}{n !} .
$$

For $k \in \mathbb{N}$ and $i_{1}, \cdots, i_{k}, n \in \mathbb{N}^{*}$, we write

$$
\begin{aligned}
{\left[f_{i_{1}}\left(x_{1}\right)\right.} & \left.+\cdots+f_{i_{k}}\left(x_{k}\right)\right]^{n} \\
& =\sum_{\substack{l_{1}+\cdots+l_{k}=n \\
\left(l_{1}, \cdots, l_{k} \geqq 0\right)}}\left(\begin{array}{c}
n \\
l_{1}, \cdots, l_{k}
\end{array}\right) f_{i_{1}+l_{1}}\left(x_{1}\right) \cdots f_{i_{k}+l_{k}}\left(x_{k}\right),
\end{aligned}
$$

where $f_{i_{j}}\left(x_{j}\right)(1 \leqq j \leqq k)$ is a sequence of polynomials. The case when $f_{n}(x)=B_{n}(x)$ in (7) was first studied by Agoh and Dilcher [14] who proved an existence theorem and also derived some explicit expressions for $k=3$ involving the Bernoulli polynomials. We now state the following higher-order convolution for the general Apostol-type polynomials $\mathcal{Y}_{n, \beta}(x ; \kappa, a, b)$ defined by (5).

Theorem 1. Let $d$ be a positive integer and let

$$
y=x_{1}+\cdots+x_{d}
$$

Then, for an integer $\kappa$ and for $m, n \in \mathbb{N}^{*}$, 


$$
\begin{gathered}
\sum_{\substack{i_{1}+\cdots+i_{d}=m \\
\left(i_{1}, \cdots, i_{d} \geqq 0\right)}}\left(\begin{array}{c}
m \\
i_{1}, \cdots, i_{d}
\end{array}\right)\left[\mathcal{Y}_{i_{1}, \beta}\left(x_{1} ; \kappa, a, b\right)+\cdots+\mathcal{Y}_{i_{d}, \beta}\left(x_{d} ; \kappa, a, b\right)\right]^{n} \\
=\left(\frac{2^{1-\kappa}}{a^{b}}\right)^{d-1} \frac{(m+n) !}{(d-1) !} \sum_{i=1}^{d} s(d, i) \sum_{j=0}^{i-1} j ! \cdot\left(\begin{array}{c}
i-1 \\
j
\end{array}\right)\left(\begin{array}{c}
\kappa+j-1 \\
j
\end{array}\right) \\
\cdot \sum_{l=0}^{i-1-j}\left(\begin{array}{c}
i-1-j \\
l
\end{array}\right) \frac{(-1)^{l} y^{i-1-j-l}}{(m+n+j-(d-1) \kappa) !} \\
\cdot \mathcal{Y}_{m+n+j+l-(d-1) \kappa, \beta}(y ; \kappa, a, b) .
\end{gathered}
$$

We first deduce some special cases of Theorem 1. By taking

$$
\alpha=1, \beta=\lambda, \kappa=0, a=-1 \text { and } b=1
$$

in (5), we have

$$
\mathcal{Y}_{n, \lambda}(x ; 0,-1,1)=\mathcal{E}_{n}(x ; \lambda) \quad\left(n \in \mathbb{N}^{*}\right) .
$$

Thus, by applying (8) to Theorem 1, we get the following higher-order convolution for the Apostol-Euler polynomials.

Corollary 1. Let $d$ be a positive integer and let

$$
y=x_{1}+\cdots+x_{d} .
$$

Then, for $m, n \in \mathbb{N}^{*}$,

$$
\begin{aligned}
& \sum_{\substack{i_{1}+\cdots+i_{d}=m \\
\left(i_{1}, \cdots, i_{d} \geqq 0\right)}}\left(\begin{array}{c}
m \\
i_{1}, \cdots, i_{d}
\end{array}\right)\left[\mathcal{E}_{i_{1}}\left(x_{1} ; \lambda\right)+\cdots+\mathcal{E}_{i_{d}}\left(x_{d} ; \lambda\right)\right]^{n} \\
& =\frac{(-2)^{d-1}}{(d-1) !} \sum_{i=1}^{d} s(d, i) \sum_{l=0}^{i-1}\left(\begin{array}{c}
i-1 \\
l
\end{array}\right)(-1)^{l} y^{i-1-l} \mathcal{E}_{m+n+l}(y ; \lambda) .
\end{aligned}
$$

Obviously, in the case when $m=0$, Corollary 1 yields the following further special case for $d \in \mathbb{N}$ and $n \in \mathbb{N}^{*}$ :

$$
\begin{aligned}
& {\left[\mathcal{E}_{0}\left(x_{1} ; \lambda\right)+\cdots+\mathcal{E}_{0}\left(x_{d} ; \lambda\right)\right]^{n}} \\
& \quad=\frac{(-2)^{d-1}}{(d-1) !} \sum_{i=1}^{d} s(d, i) \sum_{l=0}^{i-1}\left(\begin{array}{c}
i-1 \\
l
\end{array}\right)(-1)^{l} y^{i-1-l} \mathcal{E}_{n+l}(y ; \lambda),
\end{aligned}
$$

which, upon setting $i \mapsto i+1$, corresponds to the following result for the Apostol-Euler polynomials due to Bayad and Kim [15] Theorem 4:

$$
\begin{aligned}
& \sum_{\substack{l_{1}+\cdots+l_{d}=n \\
\left(l_{1}, \cdots, l_{d} \geqq 0\right)}}\left(\begin{array}{c}
n \\
l_{1}, \cdots, l_{d}
\end{array}\right) \mathcal{E}_{l_{1}}\left(x_{1} ; \lambda\right) \cdots \mathcal{E}_{l_{d}}\left(x_{d} ; \lambda\right) \\
& \quad=\frac{(-2)^{d-1}}{(d-1) !} \sum_{i=0}^{d-1}(-1)^{i} s(d, i+1) \sum_{l=0}^{i}\left(\begin{array}{l}
i \\
l
\end{array}\right)(-y)^{l} \mathcal{E}_{n+i-l}(y ; \lambda) .
\end{aligned}
$$

If we change the order of the summation on the right-hand side of (9), we get 


$$
\begin{aligned}
& {\left[\mathcal{E}_{0}\left(x_{1} ; \lambda\right)+\cdots+\mathcal{E}_{0}\left(x_{d} ; \lambda\right)\right]^{n}} \\
& \quad=\frac{2^{d-1}}{(d-1) !} \sum_{l=0}^{d-1}(-1)^{d-1-l} \mathcal{E}_{n+l}(y ; \lambda) \sum_{i=l+1}^{d}\left(\begin{array}{c}
i-1 \\
l
\end{array}\right) s(d, i) y^{i-1-l} \\
& \quad=\frac{2^{d-1}}{(d-1) !} \sum_{l=0}^{d-1}(-1)^{l} \mathcal{E}_{n+d-l-1}(y ; \lambda) \sum_{i=d-l}^{d}\left(\begin{array}{c}
i-1 \\
d-1-l
\end{array}\right) s(d, i) y^{i-d+l} .
\end{aligned}
$$

In particular, upon setting $\lambda=1$ in (10), we find for $d \in \mathbb{N}$ and $n \in \mathbb{N}^{*}$ that (see, for example, ref. [19] Theorem 5)

$$
\begin{aligned}
& \sum_{\substack{l_{1}+\cdots+l_{d}=n \\
\left(l_{1}, \cdots, l_{d} \geqq 0\right)}}\left(\begin{array}{c}
n \\
l_{1}, \cdots, l_{d}
\end{array}\right) E_{l_{1}}\left(x_{1}\right) \cdots E_{l_{d}}\left(x_{d}\right) \\
& \quad=\frac{2^{d-1}}{(d-1) !} \sum_{l=0}^{d-1}(-1)^{l} E_{n+d-l-1}(y) \sum_{i=0}^{l}\left(\begin{array}{c}
d+i-l-1 \\
i
\end{array}\right) s(d, d+i-l) y^{i} .
\end{aligned}
$$

If we take $\alpha=\kappa=b=1$ in (5), we obtain the following relationships for $n \in \mathbb{N}^{*}$ :

$$
\mathcal{Y}_{n, \lambda}(x ; 1,1,1)=\mathcal{B}_{n}(x ; \lambda) \text { and } \mathcal{Y}_{n, \lambda / 2}\left(x ; 1,-\frac{1}{2}, 1\right)=\mathcal{G}_{n}(x ; \lambda) .
$$

Consequently, Theorem 1 can be applied in conjunction with (11) in order to obtain the corresponding higher-order convolutions for the Apostol-Bernoulli polynomials and the Apostol-Genocchi polynomials. We proceed now to give here some much simpler expressions for the higher-order convolutions for the Apostol-Bernoulli polynomials and the Apostol-Genocchi polynomials.

Theorem 2. Let $d \in \mathbb{N}$ and let

$$
y=x_{1}+\cdots+x_{d}
$$

Then, for $m, n \in \mathbb{N}^{*}(m+n \geqq d)$,

$$
\begin{aligned}
& \sum_{\substack{i_{1}+\cdots+i_{d}=m \\
\left(i_{1}, \cdots, i_{d} \geqq 0\right)}}\left(\begin{array}{c}
m \\
i_{1}, \cdots, i_{d}
\end{array}\right)\left[\mathcal{B}_{i_{1}}\left(x_{1} ; \lambda\right)+\cdots+\mathcal{B}_{i_{d}}\left(x_{d} ; \lambda\right)\right]^{n} \\
& \quad=\frac{(m+n) !}{(m+n-d) ! \cdot(d-1) !} \sum_{i=1}^{d} s(d, i) \sum_{j=0}^{i-1}\left(\begin{array}{c}
i-1 \\
j
\end{array}\right) \frac{(-1)^{j} y^{i-1-j}}{m+n+j+1-d} \mathcal{B}_{m+n+j+1-d}(y ; \lambda) .
\end{aligned}
$$

For $\lambda=1$, Theorem 2 reduces to the following higher-order convolution for the Bernoulli polynomials:

$$
\begin{aligned}
& \underset{\substack{i_{1}+\cdots+i_{d}=m \\
\left(i_{1}, \cdots, i_{d} \geqq 0\right)}}{ }\left(\begin{array}{c}
m \\
i_{1}, \cdots, i_{d}
\end{array}\right)\left[B_{i_{1}}\left(x_{1}\right)+\cdots+B_{i_{d}}\left(x_{d}\right)\right]^{n} \\
& =\frac{(m+n) !}{(m+n-d) ! \cdot(d-1) !} \sum_{i=1}^{d} s(d, i) \sum_{j=0}^{i-1}\left(\begin{array}{c}
i-1 \\
j
\end{array}\right) \frac{(-1)^{j} y^{i-1-j}}{m+n+j+1-d} B_{m+n+j+1-d}(y) \\
& \left(y=x_{1}+\cdots+x_{d} ; d \in \mathbb{N} ; m, n \in \mathbb{N}^{*} ; m+n \geqq d\right) .
\end{aligned}
$$

For a different expression than that given by (12) in its special case when

$$
x_{1}=\cdots=x_{d}=x,
$$

see a known result [16] Corollary 4. 
If we set $m=0$ in Theorem 2, we get

$$
\begin{aligned}
& {\left[\mathcal{B}_{0}\left(x_{1} ; \lambda\right)+\cdots+\mathcal{B}_{0}\left(x_{d} ; \lambda\right)\right]^{n}} \\
& \quad=\frac{n !}{(n-d) ! \cdot(d-1) !} \sum_{i=1}^{d}(-1)^{i-1} s(d, i) \sum_{j=0}^{i-1}\left(\begin{array}{c}
i-1 \\
j
\end{array}\right) \frac{(-y)^{i-1-j}}{n+j+1-d} \mathcal{B}_{n+j+1-d}(y ; \lambda) \\
& \quad\left(y=x_{1}+\cdots+x_{d} ; n, d \in \mathbb{N} ; n \geqq d\right) .
\end{aligned}
$$

For $r \in \mathbb{N}$ and $m, n \in \mathbb{N}^{*}$, it is known that (see, for example, [20] Theorem 1.2)

$$
\begin{array}{r}
\sum_{k=0}^{m}\left(\begin{array}{c}
m \\
k
\end{array}\right) x^{m-k} \frac{f_{n+k+r}(y)}{\langle n+k+1\rangle_{r}}-\sum_{k=0}^{n}\left(\begin{array}{c}
n \\
k
\end{array}\right)(-x)^{n-k} \frac{f_{m+k+r}(x+y)}{\langle m+k+1\rangle_{r}} \\
=\frac{(-1)^{n+1} x^{m+n+1}}{(r-1) !} \int_{0}^{1} t^{m}(1-t)^{n} f_{r-1}(x+y-x t) \mathrm{d} t,
\end{array}
$$

where $\langle\lambda\rangle_{n}$ denotes the rising factorial of order $n$ given by

$$
\langle\lambda\rangle_{0}=1 \quad \text { and } \quad\langle\lambda\rangle_{n}=\lambda(\lambda+1) \cdots(\lambda+n-1) \quad(n \in \mathbb{N} ; \lambda \in \mathbb{C}),
$$

and $\left\{f_{n}(x)\right\}_{n=0}^{\infty}$ is a sequence of polynomials generated by

$$
\sum_{n=0}^{\infty} f_{n}(x) \frac{t^{n}}{n !}=F(t) e^{\left(x-\frac{1}{2}\right) t}
$$

with $F(t)$ being a formal power series. Thus, by taking

$$
F(t)=\frac{t e^{\frac{t}{2}}}{\lambda e^{t}-1}
$$

in (15) and substituting $n-d$ for $m, i-1$ for $n, y$ for $x$ and 0 for $y$ in (14), we find (for positive integers $i, d, n$ with $n \geqq d$ ) that

$$
\begin{gathered}
\sum_{j=0}^{n-d}\left(\begin{array}{c}
n-d \\
j
\end{array}\right) y^{n-d-j} \frac{\mathcal{B}_{i+j}(\lambda)}{i+j}-\sum_{j=0}^{i-1}\left(\begin{array}{c}
i-1 \\
j
\end{array}\right)(-y)^{i-1-j} \frac{\mathcal{B}_{n+j+1-d}(y ; \lambda)}{n+j+1-d} \\
=(-1)^{i} y^{n-d+i} \int_{0}^{1} t^{n-d}(1-t)^{i-1} \mathcal{B}_{0}(y-y t) \mathrm{d} t .
\end{gathered}
$$

It is easily seen from the properties of the Beta function $\mathrm{B}(\alpha, \beta)$ and the Gamma function $\Gamma(z)$ that

$$
\begin{aligned}
\mathrm{B}(m+1, n+1) & =\int_{0}^{1} t^{m}(1-t)^{n} \mathrm{~d} t=\frac{\Gamma(m+1) \Gamma(n+1)}{\Gamma(m+n+2)} \\
& =\frac{m ! \cdot n !}{(m+n+1) !} \quad\left(m, n \in \mathbb{N}^{*}\right)
\end{aligned}
$$

Let $\delta_{1, \lambda}$ be a Kronecker symbol given by

$$
\delta_{1, \lambda}= \begin{cases}1 & (\lambda=1) \\ 0 & (\lambda \neq 1) .\end{cases}
$$

Since $\mathcal{B}_{0}(x ; \lambda)=1$ when $\lambda=1$ and $\mathcal{B}_{0}(x ; \lambda)=0$ when $\lambda \neq 1$ (see, for example, [3]), by setting

$$
\mathcal{B}_{0}(x ; \lambda)=\delta_{1, \lambda}
$$

in (16), with the help of (17), we have

$$
\begin{gathered}
\sum_{j=0}^{i-1}\left(\begin{array}{c}
i-1 \\
j
\end{array}\right)(-y)^{i-1-j} \frac{\mathcal{B}_{n+j+1-d}(y ; \lambda)}{n+j+1-d}=\sum_{j=0}^{n-d}\left(\begin{array}{c}
n-d \\
j
\end{array}\right) y^{n-d-j} \frac{\mathcal{B}_{i+j}(\lambda)}{i+j} \\
-(-1)^{i} y^{n-d+i} \delta_{1, \lambda} \frac{(n-d) ! \cdot(i-1) !}{(n-d+i) !} .
\end{gathered}
$$


We find from (13) and (18) the following formula due to Bayad and Kim [15] Theorem 5 for sums of products of the Apostol-Bernoulli polynomials:

$$
\begin{gathered}
\sum_{i_{1}+\cdots+i_{d}=n}\left(\begin{array}{c}
n \\
\left(i_{1}, \cdots, i_{d} \geqq 0\right.
\end{array}\right) \mathcal{B}_{i_{1}}\left(x_{1} ; \lambda\right) \cdots \mathcal{B}_{i_{d}}\left(x_{d} ; \lambda\right) \\
=\frac{n !}{(d-1) !} \sum_{i=1}^{d}(-1)^{i-1} s(d, i) \sum_{j=0}^{n-d} \frac{\mathcal{B}_{i+j}(\lambda)}{j ! \cdot(n-d-j) ! \cdot(i+j)} y^{n-d-j} \\
\quad+\delta_{1, \lambda} \frac{n !}{(d-1) !} \sum_{i=1}^{d} s(d, i) \frac{(i-1) !}{(n-d+i) !} y^{n-d+i} \\
\left(y=x_{1}+\cdots+x_{d} ; n, d \in \mathbb{N} ; n \geqq d\right) .
\end{gathered}
$$

Upon changing the order of the summation on the right-hand side of (13), we get

$$
\begin{aligned}
& {\left[\mathcal{B}_{0}\left(x_{1} ; \lambda\right)+\cdots+\mathcal{B}_{0}\left(x_{d} ; \lambda\right)\right]^{n}} \\
& \quad=\frac{n !}{(n-d) ! \cdot(d-1) !} \sum_{j=0}^{d-1}(-1)^{j} \frac{\mathcal{B}_{n+j+1-d}(y ; \lambda)}{n+j+1-d} \sum_{i=j+1}^{d}\left(\begin{array}{c}
i-1 \\
j
\end{array}\right) s(d, i) y^{i-1-j} \\
& \quad=\frac{(-1)^{d-1} n !}{(n-d) ! \cdot(d-1) !} \sum_{j=0}^{d-1}(-1)^{j} \frac{\mathcal{B}_{n-j}(y ; \lambda)}{n-j} \sum_{i=d-1-j}^{d-1}\left(\begin{array}{c}
i \\
d-1-j
\end{array}\right) s(d, i+1) y^{i-(d-1-j)},
\end{aligned}
$$

which, in the special case when $\lambda=1$, yields the following famous formula for the Bernoulli polynomials due to Dilcher [19] Theorem 3:

$$
\begin{gathered}
\sum_{\substack{i_{1}+\cdots+i_{d}=n \\
\left(i_{1}, \cdots, i_{d} \geqq 0\right)}}\left(\begin{array}{c}
n \\
i_{1}, \cdots, i_{d}
\end{array}\right) B_{i_{1}}\left(x_{1}\right) \cdots B_{i_{d}}\left(x_{d}\right) \\
=(-1)^{d-1}\left(\begin{array}{l}
n \\
d
\end{array}\right) d \sum_{j=0}^{d-1}(-1)^{j}\left[\sum_{i=0}^{j}\left(\begin{array}{c}
d+i-j-1 \\
i
\end{array}\right) s(d, d+i-j) y^{i}\right] \frac{B_{n-j}(y)}{n-j} \\
\left(y=x_{1}+\cdots+x_{d} ; n, d \in \mathbb{N} ; n \geqq d\right) .
\end{gathered}
$$

Let $p_{n, m}(x)$ denote a polynomial given by (see, for example [21,22])

$$
p_{n, m}(x)=\frac{(-1)^{n-m-1}}{(n-1) !} \sum_{k=m}^{n-1}\left(\begin{array}{c}
k \\
m
\end{array}\right) s(n, k+1) x^{k-m} .
$$

Then, by applying (20) and (22), we get

$$
\begin{gathered}
\sum_{\substack{i_{1}+\cdots+i_{d}=n \\
\left(i_{1}, \cdots, i_{d} \geqq 0\right)}}\left(\begin{array}{c}
n \\
i_{1}, \cdots, i_{d}
\end{array}\right) \mathcal{B}_{i_{1}}\left(x_{1} ; \lambda\right) \cdots \mathcal{B}_{i_{d}}\left(x_{d} ; \lambda\right) \\
=\frac{(-1)^{d-1} n !}{(n-d) !} \sum_{j=0}^{d-1} p_{d, d-1-j}(y) \frac{\mathcal{B}_{n-j}(y ; \lambda)}{n-j} \\
\left(y=x_{1}+\cdots+x_{d} ; n, d \in \mathbb{N} ; n \geqq d\right),
\end{gathered}
$$

which is a generalization of the following result given by Kim and Hu [22] Theorem 1.2 for the Apostol-Bernoulli numbers: 


$$
\begin{aligned}
& \sum_{\substack{i_{1}+\cdots+i_{d}=n \\
\left(i_{1}, \cdots, i_{d} \geqq 0\right)}}\left(\begin{array}{c}
n \\
i_{1}, \cdots, i_{d}
\end{array}\right) \mathcal{B}_{i_{1}}(\lambda) \cdots \mathcal{B}_{i_{d}}(\lambda) \\
& = \begin{cases}\frac{(-1)^{n+d+1} n !}{(n-d) !} \sum_{j=0}^{d-1} p_{d, d-1-j}(d) \frac{\mathcal{B}_{n-j}\left(\frac{1}{\lambda}\right)}{n-j} & (n>d) \\
n ! \cdot p_{n, 0}(n) \mathcal{B}_{1}(\lambda)-n ! \sum_{j=0}^{n-2} p_{n, n-1-j}(n) \frac{\mathcal{B}_{n-j}\left(\frac{1}{\lambda}\right)}{n-j} & (n=d) .\end{cases}
\end{aligned}
$$

Theorem 3. Let $d \in \mathbb{N}$ and let

$$
y=x_{1}+\cdots+x_{d}
$$

Then, for $m, n \in \mathbb{N}^{*}(m+n \geqq d)$,

$$
\begin{aligned}
& \sum_{\substack{i_{1}+\cdots+i_{d}=m \\
\left(i_{1}, \cdots, i_{d} \geqq 0\right)}}\left(\begin{array}{c}
m \\
i_{1}, \cdots, i_{d}
\end{array}\right)\left[\mathcal{G}_{i_{1}}\left(x_{1} ; \lambda\right)+\cdots+\mathcal{G}_{i_{d}}\left(x_{d} ; \lambda\right)\right]^{n} \\
& \quad=\frac{(-2)^{d-1} \cdot(m+n) !}{(m+n-d) ! \cdot(d-1) !} \sum_{i=1}^{d} s(d, i) \sum_{j=0}^{i-1}\left(\begin{array}{c}
i-1 \\
j
\end{array}\right) \frac{(-1)^{j} y^{i-1-j}}{m+n+j+1-d} \mathcal{G}_{m+n+j+1-d}(y ; \lambda) .
\end{aligned}
$$

In its special case when $m=0$, Theorem 3 immediately yields

$$
\begin{aligned}
& {\left[\mathcal{G}_{0}\left(x_{1} ; \lambda\right)+\cdots+\mathcal{G}_{0}\left(x_{d} ; \lambda\right)\right]^{n}} \\
& =\frac{(-2)^{d-1} \cdot n !}{(n-d) ! \cdot(d-1) !} \sum_{i=1}^{d} s(d, i) \sum_{j=0}^{i-1}\left(\begin{array}{c}
i-1 \\
j
\end{array}\right) \frac{(-1)^{j} y^{i-1-j}}{n+j+1-d} \mathcal{G}_{n+j+1-d}(y ; \lambda) \\
& \quad\left(y=x_{1}+\cdots+x_{d} ; n, d \in \mathbb{N} ; n \geqq d\right) .
\end{aligned}
$$

By a similar consideration to that for (19), we can obtain the following formula for the Apostol-Genocchi polynomials:

$$
\begin{gathered}
\sum_{\substack{i_{1}+\cdots+i_{d}=n \\
\left(i_{1}, \cdots, i_{d} \geqq 0\right)}}\left(\begin{array}{c}
n \\
i_{1}, \cdots, i_{d}
\end{array}\right) \mathcal{G}_{i_{1}}\left(x_{1} ; \lambda\right) \cdots \mathcal{G}_{i_{d}}\left(x_{d} ; \lambda\right) \\
=\frac{(-2)^{d-1} \cdot n !}{(d-1) !} \sum_{i=1}^{d}(-1)^{i-1} s(d, i) \sum_{j=0}^{n-d} \frac{\mathcal{G}_{i+j}(\lambda)}{j ! \cdot(n-d-j) ! \cdot(i+j)} y^{n-d-j} \\
\left(y=x_{1}+\cdots+x_{d} ; n, d \in \mathbb{N} ; n \geqq d\right) .
\end{gathered}
$$

By changing the order of the summation on the right-hand side of (23), we find that

$$
\begin{gathered}
\sum_{\substack{i_{1}+\cdots+i_{d}=n \\
i_{1}, \cdots, i_{d} \geqq 0}}\left(\begin{array}{c}
n \\
i_{1}, \cdots, i_{d}
\end{array}\right) \mathcal{G}_{i_{1}}\left(x_{1} ; \lambda\right) \cdots \mathcal{G}_{i_{d}}\left(x_{d}, \lambda\right) \\
=2^{d-1}\left(\begin{array}{l}
n \\
d
\end{array}\right) d \sum_{j=0}^{d-1}(-1)^{j}\left[\sum_{i=0}^{j}\left(\begin{array}{c}
d+i-j-1 \\
i
\end{array}\right) s(d, d+i-j) y^{i}\right] \frac{\mathcal{G}_{n-j}(y ; \lambda)}{n-j} \\
\left(y=x_{1}+\cdots+x_{d} ; n, d \in \mathbb{N} ; n \geqq d\right) .
\end{gathered}
$$

Finally, upon setting $\lambda=1$ in (24), gives a formula for sums of products of the Genocchi polynomials, which is analogous to (21). 


\section{Proofs of Theorems}

Before giving the proofs of Theorems 1-3, we recall the following auxiliary results which will be needed in our proofs.

Lemma 1. ([23] Theorem 3.1 and Theorem 3.2) Let $\alpha, \lambda \in \mathbb{C}$ and $n \in \mathbb{N}^{*}$. Then

$$
\frac{\partial^{n}}{\partial t^{n}}\left\{\frac{1}{1-\lambda e^{\alpha t}}\right\}=\alpha^{n} \sum_{k=1}^{n+1} \frac{(-1)^{n+k-1}}{\left(1-\lambda e^{\alpha t}\right)^{k}}(k-1) ! \cdot S(n+1, k) .
$$

Furthermore, for $n \in \mathbb{N}$,

$$
\frac{1}{\left(1-\lambda e^{\alpha t}\right)^{n}}=\sum_{k=1}^{n} \frac{(-1)^{n-k}}{(n-1) ! \cdot \alpha^{k-1}} \frac{\partial^{k-1}}{\partial t^{k-1}}\left\{\frac{1}{1-\lambda e^{\alpha t}}\right\} \cdot s(n, k) .
$$

Lemma 2. ([20] Equations (2.6) and (3.11)) Let $n \in \mathbb{N}^{*}$. Then

$$
e^{x t} \frac{\partial^{n}}{\partial t^{n}}\{F(y, t)\}=\sum_{m=0}^{\infty}\left[\sum_{k=0}^{n}\left(\begin{array}{l}
n \\
k
\end{array}\right)(-x)^{n-k} f_{m+k}(x+y)\right] \frac{t^{m}}{m !} .
$$

Moreover, for $r \in \mathbb{N}$,

$$
\begin{gathered}
e^{x t} \frac{\partial^{n}}{\partial t^{n}}\{G(y, t)\}=\sum_{m=0}^{\infty}\left[\sum_{k=0}^{n}\left(\begin{array}{l}
n \\
k
\end{array}\right)(-x)^{n-k} \frac{f_{m+k+r}(x+y)}{\langle m+k+1\rangle_{r}}\right. \\
\left.+\frac{(-1)^{n+1} x^{m+n+1}}{(r-1) !} \int_{0}^{1} t^{m}(1-t)^{n} f_{r-1}(x+y-x t) \mathrm{d} t\right] \frac{t^{m}}{m !}
\end{gathered}
$$

where

$$
\begin{gathered}
F(y, t)=\sum_{m=0}^{\infty} f_{m}(y) \frac{t^{m}}{m !}, \\
G(y, t)=\sum_{m=0}^{\infty} \frac{f_{m+r}(y)}{\langle m+1\rangle_{r}} \frac{t^{m}}{m !},
\end{gathered}
$$

and the sequence $\left\{f_{n}(x)\right\}_{n=0}^{\infty}$ is given as in Equation (15).

Proof of Theorem 1. First of all, by setting $\alpha=1$ in (25), we get

$$
\frac{1}{\left(\lambda e^{t}-1\right)^{n}}=\sum_{k=1}^{n} \frac{(-1)^{k-1}}{(n-1) !} \frac{\partial^{k-1}}{\partial t^{k-1}}\left\{\frac{1}{\lambda e^{t}-1}\right\} \cdot s(n, k) \quad(n \in \mathbb{N}),
$$

which, for $d \in \mathbb{N}$, yields

$$
\begin{aligned}
& \left(\frac{2^{1-\kappa}}{a^{b}}\right)^{d} \cdot \frac{t^{\kappa d} e^{\left(x_{1}+\cdots+x_{d}\right) t}}{\left[\left(\frac{\beta}{a}\right)^{b} e^{t}-1\right]^{d}} \\
& \quad=\left(\frac{2^{1-\kappa}}{a^{b}}\right)^{d} \cdot \sum_{i=1}^{d} \frac{(-1)^{i-1}}{(d-1) !} t^{\kappa d} e^{y t} \frac{\partial^{i-1}}{\partial t^{i-1}}\left\{\frac{1}{\left(\frac{\beta}{a}\right)^{b} e^{t}-1}\right\} \cdot s(d, i) .
\end{aligned}
$$

Let $v \in \mathbb{N}$ and let the function $\mathfrak{f}_{v}(t)$ be differentiable with respect to $t$. If we set

$$
\mathfrak{f}_{v}(t)=\frac{2^{1-\kappa} t^{\kappa}}{\beta^{b} e^{t}-a^{b}} e^{x_{v} t}
$$


then it is clear from (5) that for $l \in \mathbb{N}^{*}$,

$$
\frac{\partial^{l}}{\partial t^{l}}\left\{\mathfrak{f}_{v}(t)\right\}=\sum_{n=0}^{\infty} \mathcal{Y}_{n+l, \beta}\left(x_{v} ; \kappa, a, b\right) \frac{t^{n}}{n !}
$$

By differentiating both sides of (28) $m$ times with respect to $t$, with the help of the general Leibniz rule presented in [18] (pp. 130-133), we obtain

$$
\begin{aligned}
& \underset{\substack{i_{1}+\cdots+i_{d}=m \\
\left(i_{1}, \cdots, i_{d} \geqq 0\right)}}{\left(\begin{array}{c}
m \\
i_{1}, \cdots, i_{d}
\end{array}\right)} \frac{\partial^{i_{1}}}{\partial t^{i} 1}\left\{\mathfrak{f}_{1}(t)\right\} \cdots \frac{\partial^{i_{d}}}{\partial t^{i_{d}}}\left\{\mathfrak{f}_{d}(t)\right\} \\
& =\left(\frac{2^{1-\kappa}}{a^{b}}\right)^{d} \sum_{i=1}^{d} \frac{(-1)^{i-1}}{(d-1) !} \frac{\partial^{m}}{\partial t^{m}}\left\{t^{\kappa d} e^{y t} \frac{\partial^{i-1}}{\partial t^{i-1}}\left\{\frac{1}{\left(\frac{\beta}{a}\right)^{b} e^{t}-1}\right\}\right\} \cdot s(d, i) \text {. }
\end{aligned}
$$

We now denote by $\left[t^{n}\right] f(t)$ the coefficient of $t^{n}$ in $f(t)$ for $n \in \mathbb{N}^{*}$. Then, by making use of the operation $\left[\frac{t^{n}}{n !}\right]$ on both sides of (30) in conjunction with (29), we find that

$$
\begin{aligned}
& \underset{\sum_{i_{1}+\cdots+i_{d}=m}\left(i_{i_{1}, \cdots, i_{d}}\right)}{\left(_{1}, \cdots, i_{d} \geqq 0\right)}\left[\mathcal{Y}_{i_{1}, \beta}\left(x_{1} ; \kappa, a, b\right)+\cdots+\mathcal{Y}_{i_{d}, \beta}\left(x_{d} ; \kappa, a, b\right)\right]^{n} \\
& \quad=\left(\frac{2^{1-\kappa}}{a^{b}}\right)^{d-1} \sum_{i=1}^{d} \frac{(-1)^{i-1}}{(d-1) !} \cdot s(d, i)\left[\frac{t^{n}}{n !}\right] \frac{\partial^{m}}{\partial t^{m}}\left\{t^{\kappa d} e^{y t} \frac{\partial^{i-1}}{\partial t^{i-1}}\left\{\frac{2^{1-\kappa}}{\beta^{b} e^{t}-a^{b}}\right\}\right\} .
\end{aligned}
$$

Also, by using the Leibniz rule, we have

$$
\begin{aligned}
\frac{\partial^{i-1}}{\partial t^{i-1}} & \left\{\frac{2^{1-\kappa}}{\beta^{b} e^{t}-a^{b}}\right\} \\
& =\frac{\partial^{i-1}}{\partial t^{i-1}}\left\{\frac{2^{1-\kappa} t^{\kappa}}{\beta^{b} e^{t}-a^{b}} \cdot \frac{1}{t^{\kappa}}\right\} \\
& =\sum_{j=0}^{i-1}\left(\begin{array}{c}
i-1 \\
j
\end{array}\right) \frac{\partial^{i-1-j}}{\partial t^{i-1-j}}\left\{\frac{2^{1-\kappa} t^{\kappa}}{\beta^{b} e^{t}-a^{b}}\right\} \cdot \frac{\partial^{j}}{\partial t^{j}}\left\{\frac{1}{t^{\kappa}}\right\} \quad(i \in \mathbb{N})
\end{aligned}
$$

and

$$
\frac{\partial^{j}}{\partial t^{j}}\left\{\frac{1}{t^{\kappa}}\right\}=(-1)^{j} j ! \cdot\left(\begin{array}{c}
\kappa+j-1 \\
j
\end{array}\right) \frac{1}{t^{\kappa+j}} \quad\left(j \in \mathbb{N}^{*}\right) .
$$

It follows from the above two identities that

$$
t^{\kappa d} e^{y t} \frac{\partial^{i-1}}{\partial t^{i-1}}\left\{\frac{2^{1-\kappa}}{\beta^{b} e^{t}-a^{b}}\right\}=\sum_{j=0}^{i-1}(-1)^{j} j ! \cdot\left(\begin{array}{c}
i-1 \\
j
\end{array}\right) e^{y t} \frac{\partial^{i-1-j}}{\partial t^{i-1-j}}\left\{\frac{2^{1-\kappa} t^{\kappa}}{\beta^{b} e^{t}-a^{b}}\right\} \cdot\left(\begin{array}{c}
\kappa+j-1 \\
j
\end{array}\right) t^{(d-1) \kappa-j}
$$

If we replace $F(y, t)$ in (26) by

$$
F(0, t)=\frac{2^{1-\kappa} t^{\kappa}}{\beta^{b} e^{t}-a^{b}}=\sum_{l=0}^{\infty} \mathcal{Y}_{l, \beta}(0 ; \kappa, a, b) \frac{t^{l}}{l !},
$$

we find for $n \in \mathbb{N}^{*}$ that

$$
e^{x t} \frac{\partial^{n}}{\partial t^{n}}\left\{\frac{2^{1-\kappa} t^{\kappa}}{\beta^{b} e^{t}-a^{b}}\right\}=\sum_{l=0}^{\infty}\left[\sum_{v=0}^{n}\left(\begin{array}{l}
n \\
v
\end{array}\right)(-x)^{n-v} \mathcal{Y}_{l+v, \beta}(x ; \kappa, a, b)\right] \frac{t^{l}}{l !} .
$$


Thus, by applying (33) to (32), we obtain

$$
\begin{aligned}
& t^{\kappa d} e^{y t} \frac{\partial^{i-1}}{\partial t^{i-1}}\left\{\frac{2^{1-\kappa}}{\beta^{b} e^{t}-a^{b}}\right\} \\
&=\sum_{l=0}^{\infty} \sum_{j=0}^{i-1} \sum_{v=0}^{i-1-j} j ! \cdot\left(\begin{array}{c}
i-1 \\
j
\end{array}\right)\left(\begin{array}{c}
i-1-j \\
v
\end{array}\right)\left(\begin{array}{c}
\kappa+j-1 \\
j
\end{array}\right) \\
& \cdot(-1)^{i-1-v} y^{i-1-j-v} \mathcal{Y}_{l+v, \beta}(y ; \kappa, a, b) \frac{t^{(d-1) \kappa+l-j}}{l !}
\end{aligned}
$$

which readily yields

$$
\begin{gathered}
\frac{\partial^{m}}{\partial t^{m}}\left\{t^{\kappa d} e^{y t} \frac{\partial^{i-1}}{\partial t^{i-1}}\left\{\frac{2^{1-\kappa}}{\beta^{b} e^{t}-a^{b}}\right\}\right\}=m ! \cdot \sum_{l=0}^{\infty} \sum_{j=0}^{i-1} \sum_{v=0}^{i-1-j}(-1)^{i-1-v} j ! \\
\cdot\left(\begin{array}{c}
i-1 \\
j
\end{array}\right)\left(\begin{array}{c}
i-1-j \\
v
\end{array}\right)\left(\begin{array}{c}
\kappa+j-1 \\
j
\end{array}\right)\left(\begin{array}{c}
(d-1) \kappa+l-j \\
m
\end{array}\right) \\
\cdot y^{i-1-j-v} \mathcal{Y}_{l+v, \beta}(y ; \kappa, a, b) \frac{t^{(d-1) \kappa+l-m-j}}{l !}
\end{gathered}
$$

that is, for $n \in \mathbb{N}^{*}$,

$$
\begin{gathered}
{\left[\frac{t^{n}}{n !}\right] \frac{\partial^{m}}{\partial t^{m}}\left\{t^{\kappa d} e^{y t} \frac{\partial^{i-1}}{\partial t^{i-1}}\left\{\frac{2^{1-\kappa}}{\beta^{b} e^{t}-a^{b}}\right\}\right\}} \\
=(m+n) ! \cdot \sum_{j=0}^{i-1} \sum_{v=0}^{i-1-j}(-1)^{i-1-v} j ! \cdot\left(\begin{array}{c}
i-1 \\
j
\end{array}\right)\left(\begin{array}{c}
i-1-j \\
v
\end{array}\right)\left(\begin{array}{c}
\kappa+j-1 \\
j
\end{array}\right) \\
\cdot y^{i-1-j-v} \frac{\mathcal{Y}_{m+n+j+v-(d-1) \kappa, \beta}(y ; \kappa, a, b)}{(m+n+j-(d-1) \kappa) !} .
\end{gathered}
$$

Finally, Theorem 1 would follow by applying (34) to (31).

Proof of Theorem 2. It is easily seen from (11) and (31) that, for $d \in \mathbb{N}$ and $m, n \in \mathbb{N}^{*}$,

$$
\begin{aligned}
& \left.\sum_{\substack{i_{1}+\cdots+i_{d}=m \\
\left(i_{1}, \cdots, i_{d}\right.}} \geqq 0\right) \\
& \left.\quad=\sum_{i_{1}, \cdots, i_{d}}^{m}\right)\left[\mathcal{B}_{i_{1}}\left(x_{1} ; \lambda\right)+\cdots+\mathcal{B}_{i_{d}}\left(x_{d} ; \lambda\right)\right]^{n} \\
& \quad=\sum_{i=1}^{d} \frac{(-1)^{i-1}}{(d-1) !}\left[\frac{t^{n}}{n !}\right] \frac{\partial^{m}}{\partial t^{m}}\left\{t^{d} e^{y t} \frac{\partial^{i-1}}{\partial t^{i-1}}\left\{\frac{1}{\lambda e^{t}-1}\right\}\right\} \cdot s(d, i) .
\end{aligned}
$$

Since $\mathcal{B}_{0}(x ; \lambda)=1$ when $\lambda=1$ and $\mathcal{B}_{0}(x ; \lambda)=0$ when $\lambda \neq 1$, by setting

$$
\mathcal{B}_{0}(x ; \lambda)=\delta_{1, \lambda}
$$

we get

$$
\frac{1}{\lambda e^{t}-1}-\frac{\delta_{1, \lambda}}{t}=\sum_{l=0}^{\infty} \mathcal{B}_{l+1}(0 ; \lambda) \frac{t^{l}}{(l+1) !},
$$

where $\delta_{1, \lambda}$ is the Kronecker symbol. Hence, by putting $r=1$ and replacing $G(y, t)$ in (27) by

$$
G(0, t)=\frac{1}{\lambda e^{t}-1}-\frac{\delta_{1, \lambda}}{t}=\sum_{l=0}^{\infty} \mathcal{B}_{l+1}(0 ; \lambda) \frac{t^{l}}{(l+1) !},
$$


and making use of (17), we find for $n \in \mathbb{N}^{*}$ that

$$
\begin{gathered}
e^{x t} \frac{\partial^{n}}{\partial t^{n}}\left(\frac{1}{\lambda e^{t}-1}-\frac{\delta_{1, \lambda}}{t}\right)=\sum_{l=0}^{\infty}\left[\sum_{v=0}^{n}\left(\begin{array}{l}
n \\
v
\end{array}\right)(-x)^{n-v} \frac{\mathcal{B}_{l+v+1}(x ; \lambda)}{l+v+1}\right. \\
\left.+(-1)^{n+1} \delta_{1, \lambda} \frac{n ! \cdot l !}{(n+l+1) !} x^{n+l+1}\right] \frac{t^{l}}{l !}
\end{gathered}
$$

which, together with the exponential series for $e^{x t}$, yields

$$
\begin{aligned}
e^{x t} \frac{\partial^{n}}{\partial t^{n}}\left\{\frac{1}{\lambda e^{t}-1}\right\}=\sum_{l=0}^{\infty}\left[\sum_{v=0}^{n}\left(\begin{array}{l}
n \\
v
\end{array}\right)(-x)^{n-v} \frac{\mathcal{B}_{l+v+1}(x ; \lambda)}{l+v+1}\right] \frac{t^{l}}{l !} \\
+(-1)^{n} n ! \cdot \delta_{1, \lambda} \sum_{l=0}^{n} \frac{x^{l} t^{l-n-1}}{l !} .
\end{aligned}
$$

It follows from (36) that

$$
\begin{gathered}
t^{d} e^{y t} \frac{\partial^{i-1}}{\partial t^{i-1}}\left\{\frac{1}{\lambda e^{t}-1}\right\}=\sum_{l=0}^{\infty}\left[\sum_{v=0}^{i-1}\left(\begin{array}{c}
i-1 \\
v
\end{array}\right)(-y)^{i-1-v} \frac{\mathcal{B}_{l+v+1}(y ; \lambda)}{l+v+1}\right] \\
+(-1)^{i-1}(i-1) ! \cdot \delta_{1, \lambda} \sum_{l=0}^{i-1} \frac{y^{l}}{l !} t^{d+l} l ! \\
l ! \\
+(d, i \in \mathbb{N}) .
\end{gathered}
$$

If we now partially differentiate both sides of (37) $m$ times with respect to $t$, then

$$
\begin{aligned}
& \frac{\partial^{m}}{\partial t^{m}}\left\{t^{d} e^{y t} \frac{\partial^{i-1}}{\partial t^{i-1}}\left\{\frac{1}{\lambda e^{t}-1}\right\}\right\} \\
& =m ! \cdot \sum_{l=0}^{\infty}\left(\begin{array}{c}
d+l \\
m
\end{array}\right)\left[\sum_{v=0}^{i-1}\left(\begin{array}{c}
i-1 \\
v
\end{array}\right)(-y)^{i-1-v} \frac{\mathcal{B}_{l+v+1}(y ; \lambda)}{l+v+1}\right] \frac{t^{d+l-m}}{l !} \\
& \quad+(-1)^{i-1}(i-1) ! \cdot m ! \cdot \delta_{1, \lambda} \sum_{l=0}^{i-1}\left(\begin{array}{c}
d+l-i \\
m
\end{array}\right) \frac{y^{l}}{l !} t^{d+l-i-m}
\end{aligned}
$$

which, for $m, n \in \mathbb{N}^{*}(m+n \geqq d)$, yields

$$
\begin{aligned}
{\left[\frac{t^{n}}{n !}\right] } & \frac{\partial^{m}}{\partial t^{m}}\left\{t^{d} e^{y t} \frac{\partial^{i-1}}{\partial t^{i-1}}\left\{\frac{1}{\lambda e^{t}-1}\right\}\right\} \\
& =\frac{(m+n) !}{(m+n-d) !} \sum_{v=0}^{i-1}\left(\begin{array}{c}
i-1 \\
v
\end{array}\right)(-y)^{i-1-v} \frac{\mathcal{B}_{m+n+v+1-d}(y ; \lambda)}{m+n+v+1-d} .
\end{aligned}
$$

By applying (38) to (35), we are led to Theorem 2.

Proof of Theorem 3. From (11) and (31), we find for $d \in \mathbb{N}$ and $m, n \in \mathbb{N}^{*}$ that

$$
\begin{aligned}
& \sum_{\substack{i_{1}+\cdots+i_{d}=m \\
\left(i_{1}, \cdots, i_{d} \geqq 0\right)}}\left(\begin{array}{c}
m \\
i_{1}, \cdots, i_{d}
\end{array}\right)\left[\mathcal{G}_{i_{1}}\left(x_{1} ; \lambda\right)+\cdots+\mathcal{G}_{i_{d}}\left(x_{d} ; \lambda\right)\right]^{n} \\
& \quad=(-2)^{d-1} \cdot \sum_{i=1}^{d} \frac{(-1)^{i-1}}{(d-1) !}\left[\frac{t^{n}}{n !}\right] \frac{\partial^{m}}{\partial t^{m}}\left\{t^{d} e^{y t} \frac{\partial^{i-1}}{\partial t^{i-1}}\left\{\frac{2}{\lambda e^{t}+1}\right\}\right\} \cdot s(d, i) .
\end{aligned}
$$

Since (see, for example, [2])

$$
\mathcal{G}_{0}(x ; \lambda)=0,
$$

by applying (3) we have

$$
\frac{2}{\lambda e^{t}+1}=\sum_{n=0}^{\infty} \mathcal{G}_{n+1}(0 ; \lambda) \frac{t^{n}}{(n+1) !} .
$$

Hence, by setting $r=1$ and taking

$$
G(0, t)=\frac{2}{\lambda e^{t}+1}
$$


in (27), we find for $n \in \mathbb{N}$ that

$$
e^{x t} \frac{\partial^{n}}{\partial t^{n}}\left\{\frac{2}{\lambda e^{t}+1}\right\}=\sum_{l=0}^{\infty}\left[\sum_{v=0}^{n}\left(\begin{array}{l}
n \\
v
\end{array}\right)(-x)^{n-v} \frac{\mathcal{G}_{l+v+1}(x ; \lambda)}{l+v+1}\right] \frac{t^{l}}{l !} .
$$

It follows from (40) that

$$
\begin{aligned}
& t^{d} e^{y t} \frac{\partial^{i-1}}{\partial t^{i-1}}\left\{\frac{2}{\lambda e^{t}+1}\right\} \\
& =\sum_{l=0}^{\infty}\left[\sum_{v=0}^{i-1}\left(\begin{array}{c}
i-1 \\
v
\end{array}\right)(-y)^{i-1-v} \frac{\mathcal{G}_{l+v+1}(y ; \lambda)}{l+v+1}\right] \frac{t^{d+l}}{l !},
\end{aligned}
$$

which implies, for $m \in \mathbb{N}^{*}$ and $i, d \in \mathbb{N}$, that

$$
\frac{\partial^{m}}{\partial t^{m}}\left\{t^{d} e^{y t} \frac{\partial^{i-1}}{\partial t^{i-1}}\left\{\frac{2}{\lambda e^{t}+1}\right\}\right\}=m ! \cdot \sum_{l=0}^{\infty}\left(\begin{array}{c}
d+l \\
m
\end{array}\right)\left[\sum_{v=0}^{i-1}\left(\begin{array}{c}
i-1 \\
v
\end{array}\right)(-y)^{i-1-v} \frac{\mathcal{G}_{l+v+1}(y ; \lambda)}{l+v+1}\right] \frac{t^{d+l-m}}{l !} .
$$

By making use of (41), we find for $m, n \in \mathbb{N}^{*}$ and $i, d \in \mathbb{N}$ that

$$
\begin{aligned}
{\left[\frac{t^{n}}{n !}\right] } & \frac{\partial^{m}}{\partial t^{m}}\left\{t^{d} e^{y t} \frac{\partial^{i-1}}{\partial t^{i-1}}\left\{\frac{1}{\lambda e^{t}+1}\right\}\right\} \\
& =\frac{(m+n) !}{(m+n-d) !} \sum_{v=0}^{i-1}\left(\begin{array}{c}
i-1 \\
v
\end{array}\right)(-y)^{i-1-v} \frac{\mathcal{G}_{m+n+v+1-d}(y ; \lambda)}{m+n+v+1-d} .
\end{aligned}
$$

Finally, by applying (42) to (39), we conclude the proof of Theorem 3.

\section{Conclusions and Observation}

In the paper, we have given a systematic and unified investigation for the Apostol-Bernoulli polynomials, the Apostol-Euler polynomials and the Apostol-Genocchi polynomials. By applying the generating-function methods and summation-transform techniques, we have established some higher-order convolutions for the Apostol-Bernoulli polynomials, the Apostol-Euler polynomials and the Apostol-Genocchi polynomials.

The methods shown in this paper may be applied to other families of special polynomials. In a similar way, some results may be obtained.

Author Contributions: All authors contributed equally.

Funding: Dr. Serkan Araci was supported by the Research Fund of Hasan Kalyoncu University in 2018.

Conflicts of Interest: The authors declare no conflict of interest.

\section{References}

1. He, Y.; Kim, T. General convolution identities for Apostol-Bernoulli, Euler and Genocchi polynomials. J. Nonlinear Sci. Appl. 2016, 9, 4780-4797.

2. Luo, Q.-M. Extension for the Genocchi polynomials and its Fourier expansions and integral representations. Osaka J. Math. 2011, 48, 291-309.

3. Luo, Q.-M.; Srivastava, H.M. Some generalizations of the Apostol-Bernoulli and Apostol-Euler polynomials. J. Math. Anal. Appl. 2005, 308, 290-302. [CrossRef]

4. Luo, Q.-M.; Srivastava, H.M. Some relationships between the Apostol-Bernoulli and Apostol-Euler polynomials. Comput. Math. Appl. 2006, 51, 631-642. [CrossRef]

5. Özarslan, M.A. Unified Apostol-Bernoulli, Euler and Genocchi polynomials. Comput. Math. Appl. 2011, 62, 2452-2462. [CrossRef] 
6. Ozden, H. Unification of generating function of the Bernoulli, Euler and Genocchi numbers and polynomials. AIP Conf. Proc. 2010, 1281, 1125-1128.

7. Ozden, H.; Simsek, Y.; Srivastava, H.M. A unified presentation of the generating functions of the generalized Bernoulli, Euler and Genocchi polynomials. Comput. Math. Appl. 2010, 60, 2779-2787. [CrossRef]

8. Lu, D.-Q.; Srivastava, H.M. Some series identities involving the generalized Apostol type and related polynomials. Comput. Math. Appl. 2011, 62, 3591-3602. [CrossRef]

9. Luo, Q.-M. Elliptic extensions of the Apostol-Bernoulli and Apostol-Euler polynomials. Appl. Math. Comput. 2015, 261, 156-166. [CrossRef]

10. Bayad, A.; Navas, L. Möbius inversion formulas related to the Fourier expansions of two-dimensional Apostol-Bernoulli polynomials. J. Number Theory 2016, 163, 457-473. [CrossRef]

11. Luo, Q.-M.; Srivastava, H.M. Some generalization of the Apostol-Genocchi polynomials and the Stirling numbers of the second kind. Appl. Math. Comput. 2011, 217, 5702-5728. [CrossRef]

12. Navas, L.M.; Ruiz, F.J.; Varona, J.L. Asymptotic estimates for Apostol-Bernoulli and Apostol-Euler polynomials. Math. Comput. 2011, 81, 1707-1722. [CrossRef]

13. Ozden, H.; Simsek, Y. Modification and unification of the Apostol-type numbers and polynomials and their applications. Appl. Math. Comput. 2014, 235, 338-351. [CrossRef]

14. Agoh, T.; Dilcher, K. Higher-order recurrences for Bernoulli numbers. J. Number Theory 2009, 129, $1837-1847$. [CrossRef]

15. Bayad, A.; Kim, T. Higher recurrences for Apostol-Bernoulli-Euler numbers. Russian J. Math. Phys. 2012, 19, 1-10. [CrossRef]

16. Bayad, A.; Komatsu, T. Zeta functions interpolating the convolution of the Bernoulli polynomials. Integral Tranforms Spec. Funct. 2018, 29, 611-622.

17. He, Y.; Kim, T. A higher-order convolution for Bernoulli polynomials of the second kind. Appl. Math. Comput. 2018, 324, 51-58. [CrossRef]

18. Comtet, L. Advanced Combinatorics: The Art of Finite and Infinite Expansions; D. Reidel Publishing Company: Dordrecht, The Netherlands, 1974.

19. Dilcher, K. Sums of products of Bernoulli numbers. J. Number. Theory 1996, 60, 23-41. [CrossRef]

20. He, Y.; Zhang, W.-P. Some symmetric identities involving a sequence of polynomials. Electron. J. Combin. 2010, 17, 1-7.

21. Kim, M.-S. A note on sums of products of Bernoulli numbers. Appl. Math. Lett. 2011, 24, 55-61. [CrossRef]

22. Kim, M.-S.; Hu, S. Sums of products of Apostol-Bernoulli numbers. Ramanujan J. 2012, 28, 113-123. [CrossRef]

23. Xu, A.-M.; Cen, Z.-D. Some identities involving exponential functions and Stirling numbers and applications. J. Comput. Appl. Math. 2014, 260, 201-207. [CrossRef] 